\title{
Radiation Transport Modeling And Assessment To Better Predict Radiation Exposure, Dose, And Toxicological Effects To Human Organs On Long Duration Space Flights
}

\author{
$\underline{\text { Pamela Denkins }}{ }^{1}$, Gautam Badhwar*2, Victor Obot $^{3}$ \\ ' Johnson Space Center, Systems Engineering and Technology Assessment, Houston, TX 77058 \\ ${ }^{2}$ Johnson Space Center, Earth Science and Solar System Exploration, Houston, TX 77058 \\ ${ }^{3}$ Texas Southern University, Department of Mathematics, Houston, TX 77004
}

\begin{abstract}
NASA's long-range plans include possible human exploratory missions to the moon and Mars within the next quarter century. Such missions beyond low Earth orbit will expose crews to transient radiation from solar particle events which include high-energy galactic cosmic rays and high-energy protons. Because the radiation levels in space are high and the missions long, adequate shielding is needed to minimize the deleterious health effects of exposure to radiation.

The focus of this study is radiation exposure to the blood-forming organs of the NASA astronauts. NASA/JSC developed the Phantom Torso Experiment for Organ Dose Measurements which housed active and passive dosimeters that would monitor and record absorbed radiation levels at vital organ locations. This experiment was conducted during the STS-91 mission in May '98 and provided the necessary space radiation data for correlation to results obtained from the current analytical models used to predict exposure to the blood-forming organs. Numerous models (i.e., BRYNTRN and HZETRN) have been developed and used to predict radiation exposure. However, new models are continually being developed and evaluated. The Space Environment Information Systems (SPENVIS) modeling program, developed by the Belgian Institute for Space Aeronomy, is to be used and evaluated as a part of the research activity. It is the intent of this research effort to compare the modeled data to the findings from the STS-91 mission; assess the accuracy and efficiency of this model; and to determine its usefulness for predicting radiation exposure and developing better guidelines for shielding requirements for long duration manned missions.
\end{abstract}

This presentation will serve to provide an overview of the research design and progress-to-date on the research activity. 